\title{
Ginsenoside Rg3 suppresses ovarian cancer cell proliferation and invasion by inhibiting the expression of IncRNA H19
}

\author{
Lingqin Zhao ${ }^{1,2 \#}$, Wei Sun ${ }^{1,2 \#}$, Aiwen Zheng ${ }^{1,2}$, Yingli Zhang ${ }^{1,2}$, Chenyan Fang ${ }^{1,2}$ and \\ Ping Zhang ${ }^{1,2}$
}

'Department of Gynecologic Oncology, Cancer Hospital of the University of Chinese Academy of Sciences, Zhejiang Cancer Hospital, Gongshu District, Hangzhou City, Zhejiang Province, 310000, China; Institute of Cancer and Basic Medicine, IBMC, Chinese Academy of Sciences, China

\begin{abstract}
Ovarian cancer $(\mathrm{OC})$ is the most malignant disease of the female reproductive system and accounts for a large proportion of gynecological cancer-related deaths. Emerging evidence has indicated that ginsenoside $\mathrm{Rg} 3$, one of the tetracyclic triterpenoid saponins in ginseng, plays crucial roles in regulating cancer progression, yet its role and mechanisms in regulating the proliferation and invasion of $O C$ are still elusive. In this study, the cell viability, proliferation, migration and invasion of $\mathrm{OC}$ were assessed by using methyl thiazol tetrazolium (MTT), colony formation, wound healing and Transwell assays, respectively. The protein levels of E-cadherin and $\mathrm{N}$-cadherin were analyzed by Western blot assay. The expression of long noncoding RNA (IncRNA) H19 was analyzed by quantitative real-time polymerase chain reaction (RT-qPCR). The results revealed that ginsenoside Rg3 significantly inhibited the viability of OC cells (SKOV3 and A2780) in a concentration-dependent manner. Ginsenoside Rg3 $(50 \mu \mathrm{g} / \mathrm{ml})$ had almost no significant effect on the activity of human ovarian epithelial cells (HOSEpiCs). Thus, this dose was selected for the subsequent experiments. Furthermore, Rg3 markedly decreased the colony formation, migration and invasion of $\mathrm{OC}$ cells. In addition, the expression of $\mathrm{N}$-cadherin was downregulated, and the expression of E-cadherin was upregulated with Rg3 treatment. Moreover, IncRNA H19 was upregulated in $\mathrm{OC}$ cells, and $\mathrm{Rg} 3$ negatively regulated H19 expression in a concentration-dependent manner. In terms of the mechanism, knockdown of $\mathrm{H} 19$ inhibited cell proliferation, migration and invasion, while overexpression of $\mathrm{H} 19$ reversed the inhibitory effect of $\mathrm{Rg} 3$ on the $\mathrm{OC}$ cells. In conclusion, ginsenoside $\mathrm{Rg} 3$ suppresses the proliferation, migration and invasion of $O C$ cells by partially inhibiting the expression of IncRNA H19.
\end{abstract}

Keywords: ovarian cancer, ginsenoside Rg3, IncRNA H19, proliferation, migration, invasion

Received: 15 April, 2020; revised: 26 July, 2020; accepted: 08 September, 2020; available on-line: 26 May, 2021

⿶e-mail: ping725020@163.com

\#The two authors have contributed equally to this article Acknowledgements of Financial Support: The research was supported by the 2018 Zhejiang Provincial Fund for Outstanding Young Talents in Traditional Chinese Medicine (No. 2018ZQ009) and Zhejiang Province Science and Technology Project of Traditional Chinese Medicine (No. 2021ZB039).

Abbreviations: IncRNA, long noncoding RNA; MT, methyl thiazol tetrazolium; OC, ovarian cancer

\section{INTRODUCTION}

Ovarian cancer is the most malignant tumor of the female reproductive system and is the 7 th most common cancer diagnosis and the 8th leading cause of cancerrelated death in women (Coburn et al., 2017; Bookman, 2016; Yokoi et al., 2018); its early symptoms are nonspecific, the disease progresses rapidly, and the 5-year survival rate is only approximately $25 \%$, which seriously threatens women's health (Pisanic et al., 2018; Zheng et al., 2019). In 2018, 22240 new ovarian cancer cases were estimated in the United States $(2.5 \%$ of all malignancies in females), and there were approximately 14070 ovarian cancer-related deaths; less than $40 \%$ of ovarian cancer patients can achieve long-term relief and cure (Torre et al., 2018). At present, the main clinical treatment method for ovarian cancer is debulking surgery combined with chemotherapy, but chemotherapeutic drugs are largely toxic and have side effects, and the overall treatment effect is not satisfactory (Berns et al., 2012; Cortez et al., 2018). Therefore, the identification of active ingredients from natural medicinal plants with low toxicity and highly effective anti-ovarian cancer activity is urgently necessary.

Ginsenoside $\operatorname{Rg} 3$ is a tetracyclic triterpenoid saponin component in ginseng. Studies have shown that ginsenoside $\operatorname{Rg} 3$ can inhibit tumor cell proliferation, invasion and metastasis, epithelial-mesenchymal transition, stemness and neovascularization, induce tumor cell apoptosis, and reverse multidrug resistance of tumor cells (Lee et al., 2015; Li et al., 2015; Li et al., 2016; Phi et al., 2019; Tian et al., 2016; Yuan et al., 2017; Wang et al., 2018). In recent years, related studies suggested the antiovarian cancer effect of ginsenoside $\mathrm{Rg} 3$ (Zheng et al., 2017; Zheng et al., 2018; Liu et al., 2017; Li et al., 2017). Interestingly, our previous results revealed that ginsenoside $\operatorname{Rg} 3$ inhibited cell proliferation, migration and invasion but promoted apoptosis in SKOV3 ovarian cancer cells (Zhao et al., 2019). Moreover, ginsenoside Rg3 decreased hypermethylation of $\mathrm{CpG}$ islands in the promoter region of tumor suppressor genes (p53, p16 and hMLH1), reduced the expression levels of DNA cytosine methyltransferases (DNMT1, DNMT3a and DNMT3b), and inhibited the activities of histone deacetylases (HDACs) (Zhao et al., 2019). All of these findings suggested that ginsenoside $\mathrm{Rg} 3$ may have potential anticancer activity in OC. The regulation of ovarian cancer cells by ginsenoside $\operatorname{Rg} 3$ through epigenetics and its specific molecular mechanism deserves further study.

Long noncoding RNA (lncRNA) is a type of RNA with transcripts longer than $200 \mathrm{nt}$. With the development of genome-wide sequencing technology and the rapid advancement of bioinformatics, the research found that lncRNAs can participate in many important regulatory processes, such as $\mathrm{X}$ chromosome silencing, genomic imprinting, chromatin modification, and transcriptional 
activation/repression. LncRNAs play an important role in the occurrence and development of many diseases, such as cancer, neurodegenerative diseases and other major diseases that seriously endanger human health (Khorkova et al., 2015). LncRNA H19, a long noncoding RNA with a length of approximately $2.6 \mathrm{~kb}$, has been shown to promote the development of cancer, including OC (Zhao et al., 2019; Wu et al., 2019). LncRNA H19 was recently identified as a crucial regulator of methylation levels of genomic DNA, mainly through interaction with S-adenosyl-homocysteine hydrolase (SAHH). Ginsenoside $\mathrm{Rg} 3$ was found to reduce the expression level of DNMT3b (Zhao et al., 2019), and and it was reported that the knockout of H19 gene activated SAHH and increased the methylation of DNMT3b mediated lncRNA H19 encoding gene Nctc1 (Zhou et al., 2015). Moreover, ginsenoside Rg3 was recently found to upregulate lncRNA H19 expression (Zheng et al., 2018), we speculated that H19 might be involved in the effects of $\mathrm{Rg} 3$ on OC.

In the present study, we explored the anticancer activity of ginsenoside $\mathrm{Rg} 3$ in OC cell lines (SKOV3 and A2780). Our results indicated that ginsenoside $\operatorname{Rg} 3$ can inhibit the proliferation, migration and invasion of OC cells. Additionally, we observed an upregulation of H19 in OC cells compared to normal controls. Rg3 negatively regulated H19 expression, and we explored the potential mechanism of this effect. This study provided a novel mechanism for the efficacy of ginsenoside $\mathrm{Rg} 3$ against $\mathrm{OC}$ and showed that ginsenoside $\mathrm{Rg} 3$ may serve as an effective drug for OC and other diseases.

\section{MATERIALS AND METHODS}

\section{Cell culture and treatment}

A human ovarian epithelial cell line (HOSEpiC) and human OC cell lines (SKOV3 and A2780) were obtained from the Type Culture Collection of the Chinese Academy of Sciences, Shanghai, China. The cells were cultured in Dulbecco's Modified Eagle's Medium (DMEM) containing 10\% fetal bovine serum (FBS) and antibiotics: penicillin plus streptomycin. The cells were cultured in a $5 \% \mathrm{CO}_{2}$ incubator at $37^{\circ} \mathrm{C}$. Ginsenoside $\operatorname{Rg} 3(\geq 98 \%$, CAS No. 14197-60-5) was purchased from MedChemExpress (Monmouth Junction, New Jersey), dissolved in PBS and then diluted in DMEM to a concentration of 1 $\mathrm{mg} / \mathrm{ml}$ which served as a stock solution. The cells were treated with ginsenoside $\mathrm{Rg} 3$ diluted to concentrations in the range of $0-800 \mu \mathrm{g} / \mathrm{ml}$ for $24 \mathrm{~h}$.

\section{Cell transfection}

The H19 fragment was amplified using PCR and then introduced into the pcDNA3.1 vector (Invitrogen, California, USA) for overexpression of lncRNA H19, and pcDNA3.1 empty vector was used as a control (pcDNA). For knockdown of H19, shRNA targeting lncRNA H19 and negative control (NC) shRNA were purchased from GeneCopoeia (Guangzhou, China). The sequences were as follows: sh-H19, 5'-CGTGACAAGCAGGACATGA-3'; sh-NC, 5'-T'TCTCCGAACGTGTCACGT-3'. The plasmids were introduced into cells by transient transfection with Lipofectamine 2000 (Invitrogen, Carlsbad, CA, USA) in accordance with the manufacturer's instructions.

\section{Cell viability assay}

The cell viability was measured by MT'T (Sigma-Aldrich, Merck KGaA, Darmstadt, Germany) assay. Cells were seeded into 96 -well plates $\left(1 \times 10^{5}\right.$ cells/well), treated with ginsenoside $\operatorname{Rg} 3$ at different concentrations and cultured at $37^{\circ} \mathrm{C}$ in a $5 \% \mathrm{CO}_{2}$ incubator for $24 \mathrm{~h}$. Then, $10 \mu \mathrm{l}$ of the MTT solution (Beyotime, Shanghai, China) was added to the cells and incubated for $4 \mathrm{~h}$. The optical density (OD) values were read at $490 \mathrm{~nm}$ using a microplate reader (Bio-Rad, Hercules, CA).

\section{Colony formation assay}

Cells (500 per well) transfected as required were seeded into 6-well plates and exposed to ginsenoside $\mathrm{Rg} 3$ for $24 \mathrm{~h}$ following transfection. After culturing for 14 days, the colonies were fixed with $4 \%$ paraformaldehyde for $15 \mathrm{~min}$ and stained with $0.5 \%$ crystal violet (Sigma) for $30 \mathrm{~min}$. The colonies were photographed using a light microscope (TE2000-U, Nikon, Japan).

\section{Wound healing assay}

Cells $\left(10^{4}\right.$ per well $)$ transfected as indicated were seeded into 6-well plates, cultured to reach $100 \%$ confluence and then exposed to ginsenoside $\operatorname{Rg} 3$ for $24 \mathrm{~h}$. A straight scratch was made with a pipette tip in each well. The wounds were photographed at $0 \mathrm{~h}$ and $24 \mathrm{~h}$ using a microscope (Nikon) and the width of the wound was measured.

\section{Cell invasion assay}

Cells $\left(1 \times 10^{5}\right.$ per well $)$ resuspended in $200 \mu \mathrm{l}$ serumfree medium were seeded into the top chamber of an insert (Corning Costar Co., Cambridge, MA, USA) precoated with Matrigel. Then, $800 \mu$ l of the medium containing 10\% FBS was added into the lower chamber. After $48 \mathrm{~h}$, the cells on the membrane were fixed and stained using crystal violet (Takara, Dalian, China).

\section{RT-qPCR}

Total RNA was isolated using TRIzol reagent (Invitrogen, Carlsbad, CA, USA) according to the manufacturer's instructions. The RNA was then reverse transcribed into cDNA using the PrimeScript RT reagent Kit with gDNA Eraser (Takara Bio, Otsu, Japan). mRNA expression was examined using the ABI PRISM 7300 RealTime PCR System (Applied Biosystems, Foster City, CA, USA) and the ExScript SYBR green QPCR kit (TaKaRa, Dalian, China). The relative mRNA expression was normalized to the expression of GAPDH using the 2- $\Delta \Delta C T$ method. The primers were as follows: lncRNA H19 (forward): 5'-ACCACTGCACTACCTGACTC-3', (reverse): 5'-CCGCAGGGGGTGGCCATGAA-3'; GAPDH (forward): 5'-CATGTACGTTGCTATCCAGGC-3', (reverse): 5'-CTCCTTAATGTCACGCACGAT-3'.

\section{Western blot analysis}

Cells were harvested and lysed in RIPA buffer supplemented with protease inhibitors $(50 \mathrm{mM}$ Tris- $\mathrm{HCl} \mathrm{pH}$ $8,50 \mathrm{mM} \mathrm{NaCl}, 0.5 \% \mathrm{NP}-40)$. The protein concentration was determined by the Bio-Rad (Bradford) protein assay (Bio-Rad, Hercules, CA, USA), and $50 \mu \mathrm{g}$ samples of total protein were separated by denaturing $12 \%$ SDSPAGE and transferred onto a PVDF membrane (Amersham, Little Chalfont, UK). After blocking with 5\% nonfat milk in a $0.1 \%$ TBST solution for $1 \mathrm{~h}$ at room temperature, the membranes were first incubated with primary antibodies (all from Abcam, Cambridge, MA, USA) against E-cadherin (ab15148; 1:1000), N-cadherin (ab76057; 1:1000), and GAPDH (ab181602, 1:1000) 

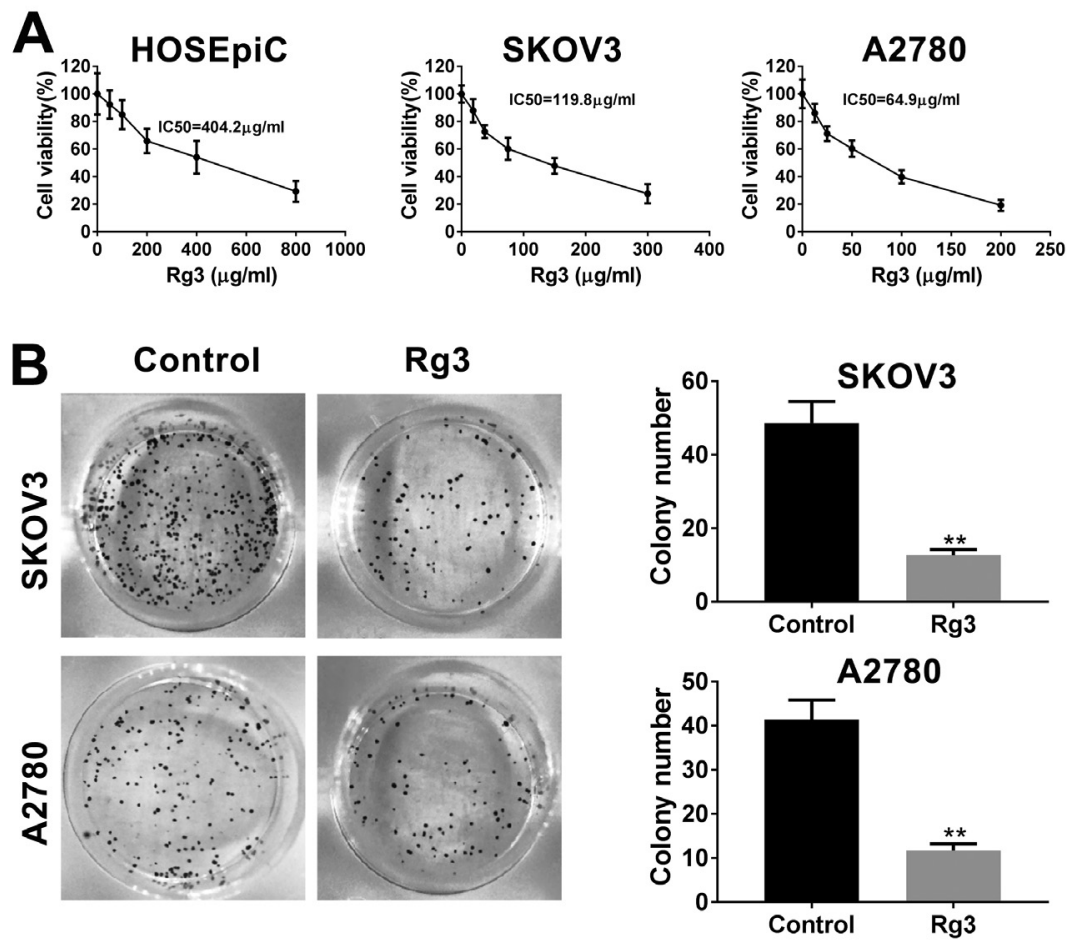

Figure 1. Ginsenoside Rg3 inhibits the proliferation of ovarian cancer cells.

(A) MTT assay was employed to determine the effect of Rg3 on the viability of HOSEpiC, SKOV3 and A2780 cells treated with Rg3. (B) Colony formation assay was used to measure the proliferation of SKOV3 and A2780 cells untreated or treated with Rg3. Data were presented as the mean \pm S.D. of at least three independent experiments. ${ }^{* *} p<0.01$.

overnight at $4^{\circ} \mathrm{C}$. After washing, blots were incubated with HRP-conjugated goat anti-rabbit (ab6721, 1:2000) or HRP-conjugated goat anti-mouse (ab205719, 1:2000) secondary antibodies for $1 \mathrm{~h}$. Immunostaining of the protein bands was visualized using an enhanced chemiluminescence (ECL) detection system. The protein levels were determined by normalization against GAPDH.

\section{Statistical analysis}

All the results were presented as the mean \pm S.D. and were analyzed using GraphPad Prism 7.0 (GraphPad Software Inc., San Diego, CA, USA) and SPSS software (IBM SPSS 22.0, Chicago, IL). Two-tailed Student's ttest and one-way ANOVA were used to compare the differences between two groups and among multiple groups, respectively. $P<0.05$ was considered as an indicator of statistical significance.

\section{RESULTS}

\section{Ginsenoside Rg3 inhibits the proliferation of ovarian cancer cells}

HoSEpiC, SKOV3 and A2780 cells were treated with different concentrations of $\operatorname{Rg} 3$ for $24 \mathrm{~h}$, and cell viability was determined by MTT assay. The data indicated that Rg3 showed almost no cytotoxicity toward HoSEpiC cells but significantly decreased the viability of SKOV3 and A2780 cells in a concentration-dependent manner with IC50 values of $119.8 \mu \mathrm{g} / \mathrm{ml}$ and $64.9 \mu \mathrm{g} /$ $\mathrm{ml}$, respectively (Fig. 1A). Therefore, the concentration of $\operatorname{Rg} 3$ used in the subsequent experiments was $50 \mu \mathrm{g} /$ $\mathrm{ml}$. Moreover, colony formation assays also confirmed that $50 \mu \mathrm{g} / \mathrm{ml} \mathrm{Rg} 3$ significantly inhibited the proliferation of SKOV3 and A2780 cells (Fig. 1B).

\section{Ginsenoside Rg3 inhibits ovarian cancer cells' migration and invasion}

Strong migration and invasion are critical steps in cancer metastasis (Yamaguchi et al., 2007). Therefore, we evaluated the effect of $\operatorname{Rg} 3$ on the migratory and invasion abilities of OC cells through wound healing and Transwell assays. The results showed that Rg3 decreased the cell migration speed and the number of invading cells in both SKOV3 and A2780 cells (Fig. 2A-B). Tumor metastasis is usually accompanied by epithelialmesenchymal transition (EMT). EMT is a physiological process in which epithelial cells lose their polarity and cell-to-cell adhesion and acquire a mesenchymal phenotype, which is characterized by upregulation of $\mathrm{N}$-cadherin and downregulation of E-cadherin (Lamouille et al., 2014). Western blot assay data indicated that Rg3 increased E-cadherin levels but decreased $\mathrm{N}$-cadherin levels in SKOV3 and A2780 cells (Fig. 2C), indicating that $\mathrm{Rg} 3$ might reverse the EMT process in OC cells.

\section{Ginsenoside Rg3 inhibits the expression of IncRNA H19 in ovarian cancer cells}

In view of the above results and recent studies showing that $\mathrm{Rg} 3$ can downregulate the expression of H19 (Zheng et al., 2018), we determined whether the inhibitory effect of $\mathrm{Rg} 3$ on $\mathrm{OC}$ cells is exerted by affecting the expression of H19. As shown in Fig. 3A, aberrantly high expression of H19 was observed in SKOV3 and A2780 cells when compared to HOSEpiC cells. However, the expression of H19 was significantly downregulated by Rg3 pretreatment at different concentrations and times 
A

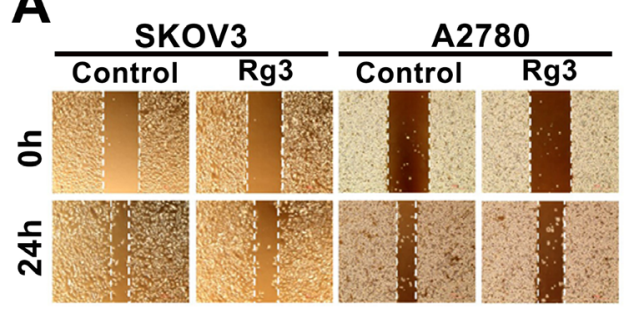

SKOV3

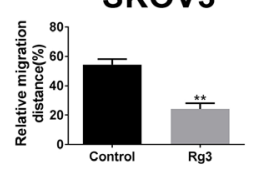

A2780

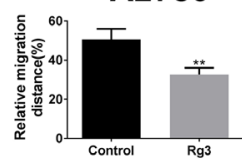

Rg3
B
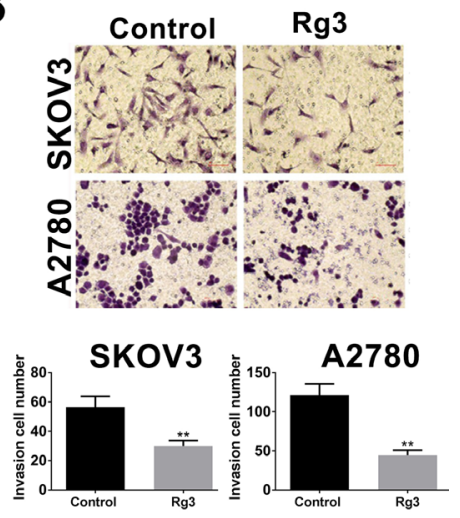

C

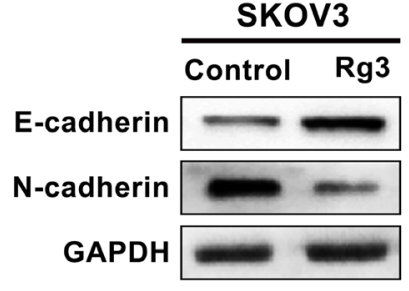

A2780

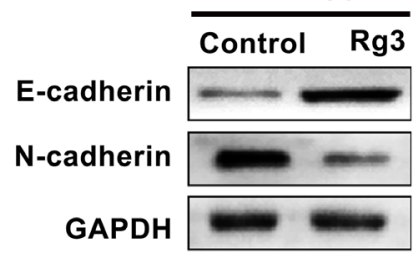

\section{.}
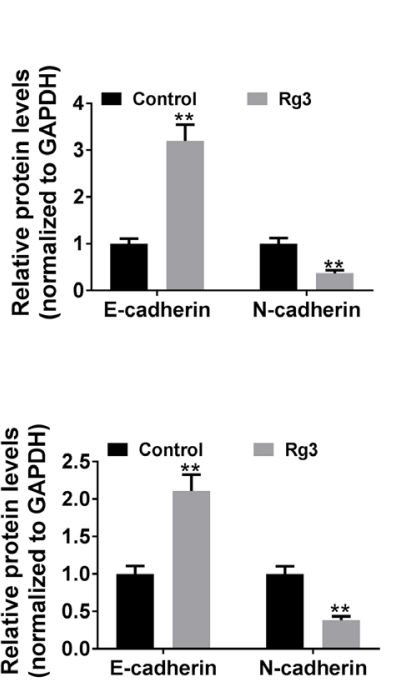

Figure 2. Ginsenoside Rg3 inhibits ovarian cancer cells' migration and invasion.

(A-B) Wound healing and Transwell assays were used to determine the effect of Rg3 on the migration and invasion of SKOV3 and A2780 cells. (C) Western blot assay was used to detect the effect of Rg3 on the protein levels of E-cadherin and N-cadherin in SKOV3 and A2780 cells. Data were presented as the mean \pm S.D. of at least three independent experiments. ${ }^{* *} p<0.01$.
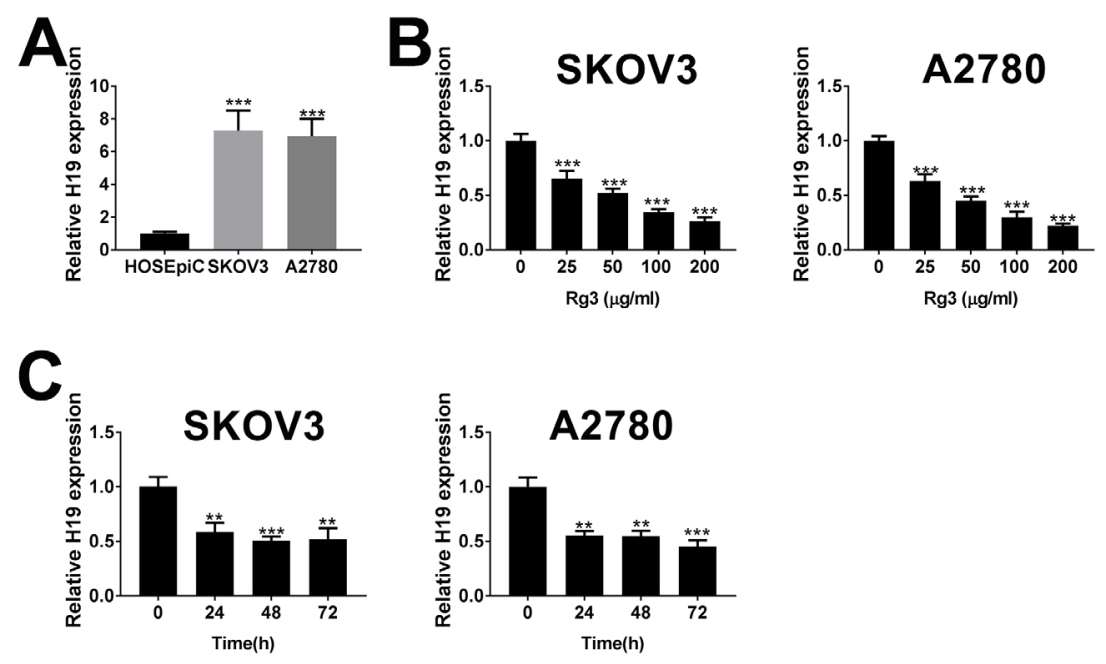

Figure 3. Ginsenoside Rg3 inhibits the expression of IncRNA H19 in ovarian cancer cells.

(A) The expression of H19 in HOSEpiC, SKOV3 and A2780 cells was measured by RT-qPCR. (B) The expression of H19 in SKOV3 and A2780 cells treated with different concentrations of Rg3 was determined by RT-qPCR. (C) The expression of H19 in SKOV3 and A2780 cells treated with Rg3 for different times was determined by RT-qPCR. Data were presented as the mean \pm S.D. of at least three independent experiments. ${ }^{* *} p<0.01,{ }^{* * *} p<0.001$. 

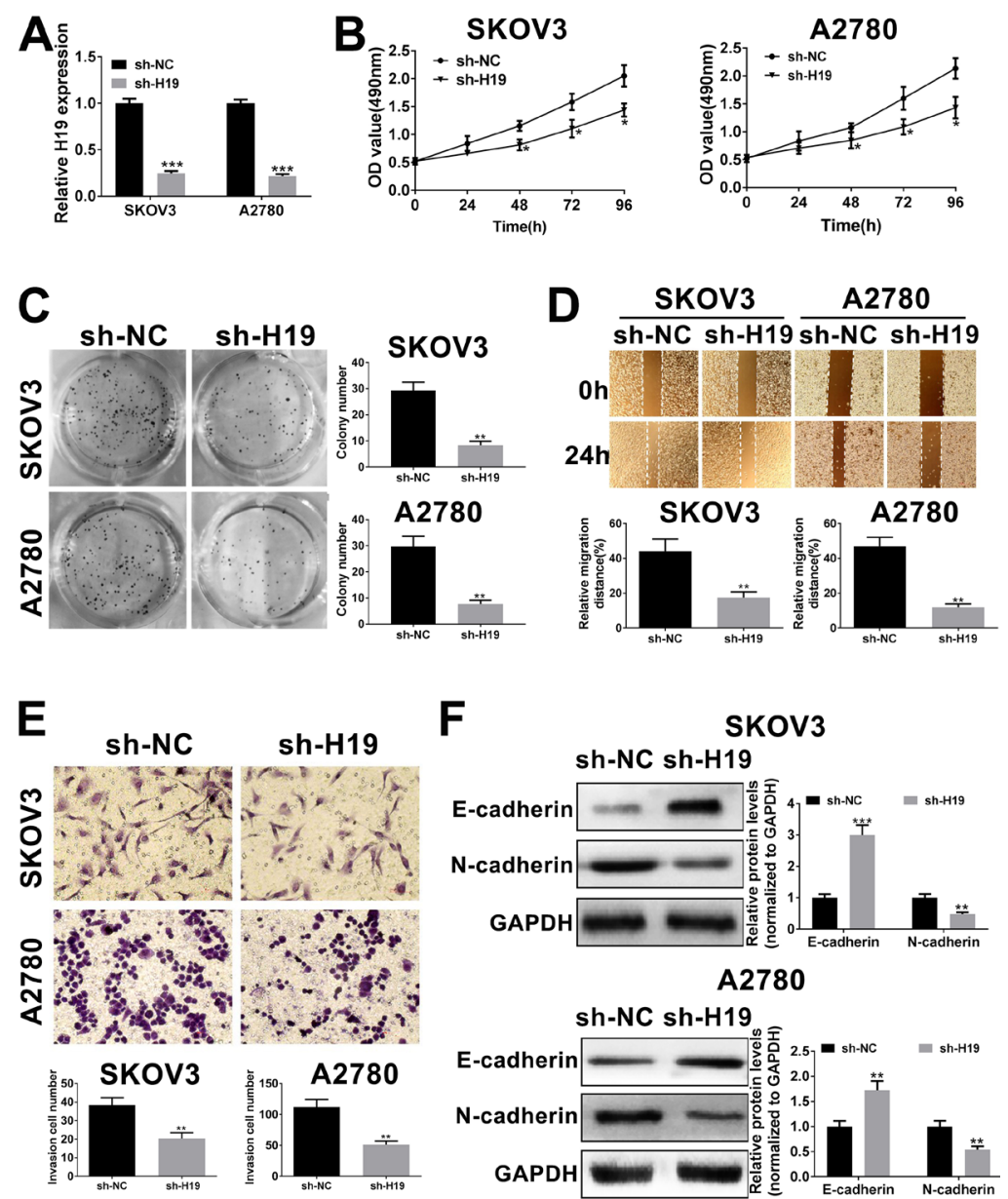

Figure 4. Downregulation of the expression of IncRNA H19 inhibits the proliferation, migration and invasion of ovarian cancer cells. (A) Satisfactory transfection efficiency was obtained at $48 \mathrm{~h}$ post-transfection with H19 in SKOV3 and A2780 cells. (B-C) MTT and colony formation assays were used to measure the effect of $\mathrm{H} 19$ on $\mathrm{OC}$ cells' proliferation. (D-E) Wound healing and Transwell assays were employed to measure the effect of H19 on OC cells' migration and invasion. (F) Western blot assay was used to detect the effect of H19 on the protein levels of E-cadherin and N-cadherin in SKOV3 and A2780 cells. Data were presented as the mean \pm S.D. of at least three independent experiments. ${ }^{*} p<0.05,{ }^{* *} p<0.01,{ }^{* * *} p<0.001$.

in a concentration-dependent manner (Fig. 3B-C), which suggests that $\mathrm{Rg} 3$ has the potential to downregulate H19 expression and that $\mathrm{H} 19$ might be involved in the anticancer effects of $\mathrm{Rg} 3$ on OC cells.

\section{Downregulation of the expression of IncRNA H19 inhibits the proliferation, migration and invasion of the ovarian cancer cells}

Numerous studies demonstrated that lncRNA H19 plays an important role in promoting ovarian cancer progression (Wu et al., 2019; Ji et al., 2019; Zheng et al., 2016). Here, we knocked down H19 by transfection with sh-H19 in SKOV3 and A2780 cells. RT-qPCR assays showed that when compared to the sh-NC group, the expression level of $\mathrm{H} 19$ in the sh-H19 group was significantly decreased, indicating that H19 was successfully knocked down in the transfected cells (Fig. 4A). Consistent with the literature research, knockdown of H19 distinctly decreased the cell viability, colony number, migration and invasion of SKOV3 and A2780 cells (Fig. 4B-E). Moreover, downregulation of lncRNA H19 expression increased E-cadherin and decreased N-cadherin expression (Fig. 4F). Taken together, these data indicate that H19 serves as an oncogene to promote cell proliferation, migration and invasion in OC progression.

\section{Ginsenoside $\mathrm{Rg} 3$ exerts its function in ovarian cancer cells at least partially through regulating $\mathrm{H} 19$}

Since $\operatorname{Rg} 3$ can negatively regulate the expression of $\mathrm{H} 19$, the anticancer mechanism of $\mathrm{Rg} 3$ may partly inhibit the oncogenic function of $\mathrm{H} 19$ by reducing its expression. To verify our hypothesis, SKOV3 and A2780 cells were transfected with pcDNA3.1-H19 to overexpress $\mathrm{H} 19$ and treated with $\mathrm{Rg} 3$ to detect the effects on cell proliferation, migration and invasion. As shown in Fig. 5A, compared to the PBS+Vector group, the expression level of H19 in the PBS+H19 group was significantly increased; that is, H19 was successfully overexpressed in the transfected cells, which was verified using RT-qPCR assay. The results indicated that overexpression of $\mathrm{H} 19$ promoted the cell proliferation, migration, invasion and EMT of OC cells compared to that in the PBS+Vector group, while the oncogenic effects of H19 were inhibited by simultaneous treatment with $\mathrm{Rg} 3$ (Fig. 5B-F). Similarly, the anticancer effect of Rg3 was also reversed by the simultaneous upregulation of H19 

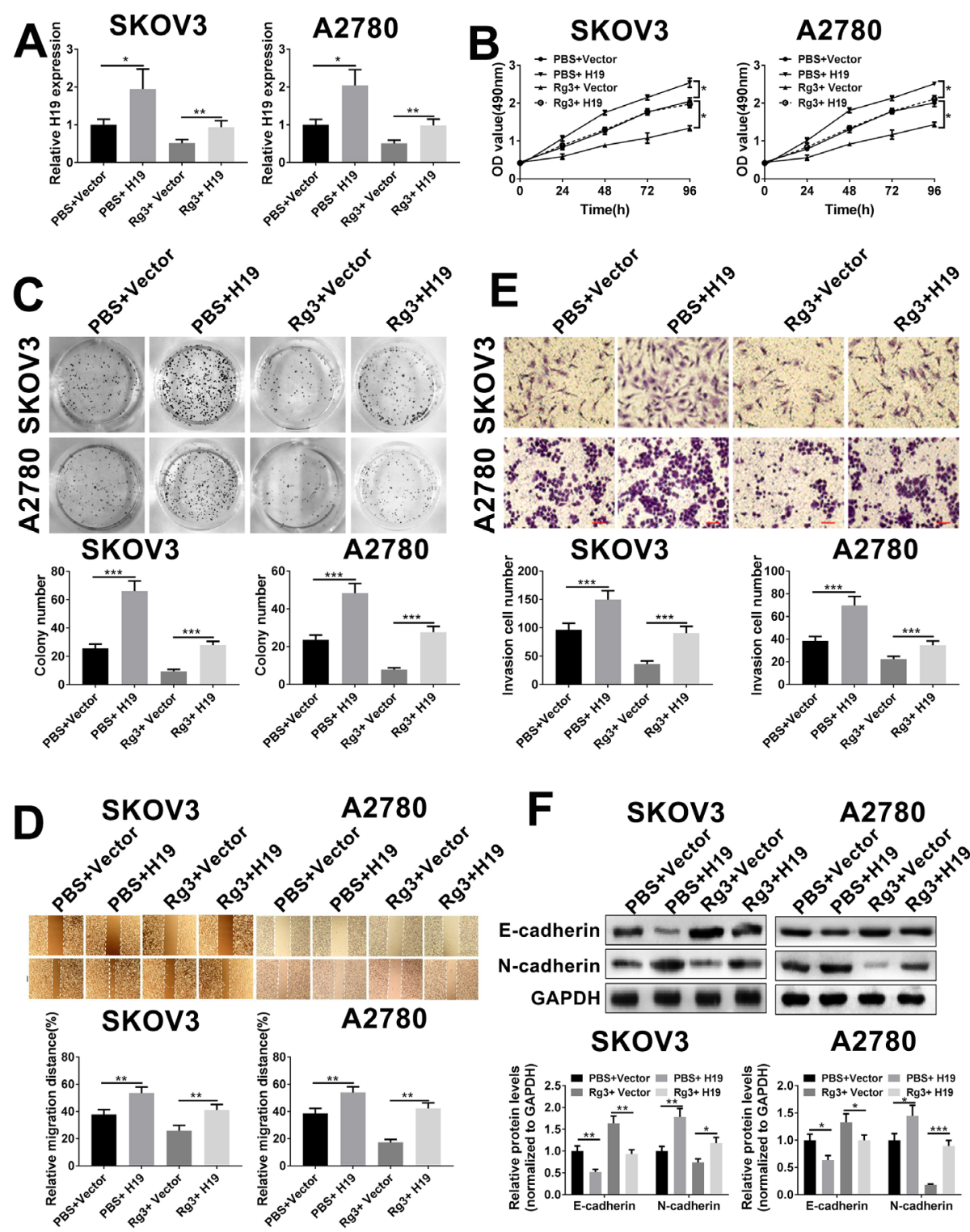

Figure 5. Ginsenoside Rg3 exerts its function in ovarian cancer cells at least partially through regulating $\mathrm{H} 19$.

(A) The expression of $\mathrm{H} 19$ in different groups was determined by RT-qPCR. (B-C) The effect of Rg3 on OC cell proliferation was reversed when cells were also transfected with $\mathrm{H} 19$ as measured by MTT and colony formation assays. (D-E) The effect of Rg3 on OC cell migration and invasion could be reversed when cells were also transfected with $\mathrm{H} 19$ as detected by wound healing and Transwell assays. (F) The effect of Rg3 on the protein levels of E-cadherin and N-cadherin in OC cells could be reversed when cells were also transfected with $\mathrm{H} 19$ as detected by Western blot assay. Data were presented as the mean \pm S.D. of at least three independent experiments. ${ }^{*} p<0.05$, ${ }^{* *} p<0.01,{ }^{* * *} p<0.001$.

(Fig. 5B-F). Therefore, Rg3 may target and negatively regulate $\mathrm{H} 19$ as part of its anticancer mechanism.

\section{DISCUSSION}

Ovarian cancer is a gynecological malignant tumor that is mainly treated by surgery combined with chemotherapy, radiation therapy, and some immunomodulators (Zheng et al., 2016). However, resistance to chemotherapeutics (carboplatin/paclitaxel doublet) often severely limits the efficacy and outcome of the treatment, even leading to a high recurrence rate (Coukos et al., 1998). Traditional Chinese herbs can increase the effectiveness of chemotherapy and reduce its side effects. Therefore, the combination of Chinese herbal medicine and chemotherapeutic drugs is a very common and promising ap- proach in the clinical treatment of cancer, especially in China and South Korea. Ginsenoside Rg3, as the main chemical component of ginseng, has been identified as a therapeutic agent for many lethal cancers (Coukos \& Rubin, 1998). In the present study, we found that ginsenoside Rg3 can specifically target OC cells and is not toxic to noncancerous cells, making it a valuable chemotherapeutic. We explored the potential of ginsenoside $\mathrm{Rg} 3$ to inhibit the proliferation, migration and invasion of OC cells, possibly by inhibiting the expression of H19.

There is growing evidence demonstrating that ginsenoside $\operatorname{Rg} 3$ participates in important cellular activities, showing anticancer properties in various cancers. Consistently, we confirmed that ginsenoside $\mathrm{Rg} 3$ inhibited OC progression by decreasing cell proliferation, migration and invasion. Moreover, for the first time, we revealed that $\operatorname{Rg} 3$ regulates the levels of EMT-related 
proteins in OC cells by increasing E-cadherin levels and decreasing $\mathrm{N}$-cadherin levels, suggesting a possible mechanism for inhibiting ovarian cancer metastasis. Usually, $\operatorname{Rg} 3$ was reported to play an antitumor role by regulating a large number of protein-coding genes or crucial cancer-related signaling pathways. Tian and others (Tian et al., 2016) revealed that ginsenoside $\mathrm{Rg} 3$ inhibits EMT and lung cancer cell invasion by inhibiting FUT4. Yuan and others (Yuan et al., 2017) reported that ginsenoside $\mathrm{Rg} 3$ promotes the cytotoxicity of paclitaxel in triplenegative breast cancer by inhibiting the $\mathrm{NF}-x \mathrm{~B}$ pathway and regulating $\mathrm{Bax} / \mathrm{Bcl}-2$ expression. Additionally, $\mathrm{Rg} 3$ was shown to inhibit PI3K-AKT (Xie et al., 2017), ERK (Tang et al., 2018), AMPK (Yuan et al., 2010) and other signaling pathways. Rg3 was also shown to be engaged in a wide range of mechanisms and have numerous targets by which it exerts its important cellular activities, but the mechanism of its lncRNA-mediated role is still poorly understood and remains to be fully elucidated.

LncRNA has no protein-coding function and is involved in regulating protein-coding genes in the form of RNA at various levels (epigenetic regulation, transcriptional regulation, post-transcriptional regulation, regulation of miRNA, etc.) (Yuan et al., 2010). Recent studies showed that abnormal changes in lncRNAs are a new mechanism of drug response regulation during tumor progression. Xiong and others (Xiong et al., ????) indicated that chronic oxymatrine therapy induces drug resistance and EMT by targeting lncRNA metastasisassociated lung adenocarcinoma transcript 1 (MALAT1) in colorectal cancer cells. Geng and others (Geng et al., ????) reported that resveratrol suppressed cell proliferation, migration and invasion by downregulating the expression of lncRNA NEAT1 in myeloma cells. In addition, the role of lncRNAs in $\mathrm{Rg} 3$-mediated regulation of tumor progression was studied. For example, $\mathrm{Rg} 3$ was confirmed to inhibit cancer progression by downregulating the expression of CCAT1 and RFX3-AS1 and upregulating the expression of STXBP5-AS1 and CASC2 (Li et al., 2019; Ham et al., 2019; Zou et al., 2020). Moreover, a new study suggested that $\operatorname{Rg} 3$ decreases the level of 67 lncRNAs in ovarian cancer, of which H19 is one of the most downregulated lncRNAs (Zheng et al., 2018). Rg3 was also confirmed to abrogate the competitive inhibition of miR-324-5p by H19, thus enhancing the inhibition of PKM2 by miR-324-5p resulting in inhibition of the Warburg effect and the occurrence of OC (Zheng et al., 2018). Nevertheless, it is still unclear whether the dysregulation of H19 expression is the molecular mechanism by which Rg3 affects the proliferation and metastasis of OC. In this study, H19 was upregulated in OC cells but downregulated by $\mathrm{Rg} 3$ treatment, which is consistent with the literature (Ji et al., 2019; Wu et al., 2019; Zheng et al., 2018, Zheng et al., 2016). Hence, a lossof-function assay was employed to investigate the effect of H19 in OC cells. We discovered that knockdown of H19 significantly inhibited cell proliferation, migration and invasion. Knockdown of $\mathrm{H} 19$ also inhibited the EMT of OC cells; that is, it increased the level of E-cadherin and decreased the level of N-cadherin. Moreover, a rescue assay was performed by simultaneous H19 overexpression and Rg3 treatment, and it was found that the tumor-suppressive function of $\mathrm{Rg} 3$ could be partially reversed by $\mathrm{H} 19$ overexpression. All these data imply that $\mathrm{Rg} 3$ exerts an anticancer effect in OC cells by negatively regulating $\mathrm{H} 19$.

Although the regulatory effect of $\operatorname{Rg} 3$ on $\operatorname{lncRNA}$ H19 was identified in the present study, the specific downstream mechanism by which Rg3 affects H19 has not been fully determined. H19 may intervene in OC pathogenesis by sponging with miR-370-3p, thus acting as a ceRNA (Li et al., 2018), or may directly affect the expression of IGF2, EZH2, SAHH and other genes to participate in cancer progression (Murphy et al., 2006; Zhou et al., 2015; Luo et al., 2013). Therefore, the mechanism by which H19 exhibits its oncogenic functions in the treatment of ovarian cancer with $\mathrm{Rg} 3$ is worth further investigation.

In conclusion, we revealed the function of $\operatorname{Rg} 3$ and for the first time demonstrated that $\operatorname{Rg} 3$ suppresses OC cell proliferation, migration and invasion by negatively regulating lncRNA H19. Our study provides preliminary confirmation of the potential use of $\mathrm{Rg} 3$ as an anticancer agent, which may represent a new nontoxic alternative to traditional cancer treatment.

\section{Acknowledgments}

Not applicable.

\section{Competing interests}

The authors state that there are no conflicts of interest to disclose.

\section{Availability of data and materials}

All data generated or analyzed during this study are included in this published article.

\section{Authors' contributions}

PZ conceived and designed the experiments; LQZ and WS analyzed and interpreted the results of the experiments; and AWZ, YLZ and CYF performed the experiments.

\section{Ethics approval and consent to participate}

Not applicable.

\section{REFERENCES}

Berns EMJJ, Bowtell DD (2012) The changing view of highgrade serous ovarian cancer. Cancer Res 72: 2701. https://doi. org/10.1158/0008-5472.CAN-11-3911

Bookman M (2016) Optimal primary therapy of ovarian cancer. Ann Oncol 27: i58-i62

Coburn SB, Bray F, Sherman ME, Trabert B (2017) International patterns and trends in ovarian cancer incidence, overall and by histologic subtype. Int J Cancer 140: 2451-2460. https://doi.org/10.1002/ ijc.30676

Cortez AJ, Tudrej P, Kujawa KA, Lisowska KM (2018) Advances in ovarian cancer therapy. Cancer Chemoth Pharmacol 81: 17-38. https:// doi.org/10.1007/s00280-017-3501-8

Coukos G, Rubin SC (1998) Chemotherapy resistance in ovarian cancer: New molecular perspectives. Obstetrics Gynecol 91: 783-792. https://doi.org/10.1016/S0029-7844(98)00054-4

Ham J, Jeong D, Park S, Kim HW, Kim H, Kim SJ (2019) Ginsenoside Rg3 and Korean Red Ginseng extract epigenetically regulate the tumor-related long noncoding RNAs RFX3-AS1 and STXBP5-AS1. J Ginseng Res 43: 625-634. https://doi.org/10.1016/j.jgr.2019.02.004

Khorkova O, Hsiao J, Wahlestedt C (2015) Basic biology and therapeutic implications of lncRNA. Adv Drug Deliv Rev 87: 15-24. https://doi.org/10.1016/j.addr.2015.05.012

Lamouille S, Xu J, Derynck R (2014) Molecular mechanisms of epithelial-mesenchymal transition. Nat Rev Mol Cell Biol 15: 178. https:// doi.org/10.1038/nrm3758

Lee SG, Kang YJ, Nam J-O (2015) Anti-metastasis effects of ginsenoside $\operatorname{Rg} 3$ in B16F10 cells. I Microbiol Biotechnol 25: 1997-2006. https://doi.org/10.4014/jmb.1506.06002

Li J, Liu T, Zhao L, Chen W, Hou H, Ye Z, Li X (2015) Ginsenoside 20 (S)-Rg3 inhibits the Warburg effect through STAT3 pathways in ovarian cancer cells. Int J Oncol 46: 775-781. https://doi. org/10.3892/ijo.2014.2767

Li J, Lu J, Ye Z, Han X, Zheng X, Hou H, Chen W, Li X, Zhao (2017) 20(S)-Rg3 blocked epithelial-mesenchymal transition through 
DNMT3A/miR-145/FSCN1 in ovarian cancer. Oncotarget 8: 5337553386. https://doi.org/10.18632/oncotarget.18482

Li J, Huang Y, Deng X, Luo M, Wang X, Hu H, Liu C, Zhong M (2018) Long noncoding RNA H19 promotes transforming growth factor- $\beta$-induced epithelial-mesenchymal transition by acting as a competing endogenous RNA of miR-370-3p in ovarian cancer cells. Onco Targets Ther 11: 427-440. https://doi.org/10.2147/OTT. S149908

Li J, Qi Y (2019) Ginsenoside Rg3 inhibits cell growth, migration and invasion in Caco-2 cells by downregulation of lncRNA CCAT1. Exp Mol Pathol 106: 131-138. https://doi.org/10.1016/j. yexmp.2019.01.003

Li Y, Yang T, Li J, Hao HL, Wang SY, Yang J, Luo JM (2016) Inhibition of multiple myeloma cell proliferation by ginsenoside Rg3 via reduction in the secretion of IGF-1. Mol Med Rep 14: 2222-2230. https://doi.org/10.3892/mmr.2016.5475

Liu T, Zhao L, Hou H, Ding L, Chen W, Li X (2017) Ginsenoside 20(S)-Rg3 suppresses ovarian cancer migration via hypoxia-inducible factor 1 alpha and nuclear factor-kappa B signals. Tumor Biol 39: https://doi.org/10.1177/1010428317692225

Luo M, Li Z, Wang W, Zeng Y, Liu Z, Qiu J (2013) Long non-coding RNA H19 increases bladder cancer metastasis by associating with EZH2 and inhibiting E-cadherin expression. Cancer Lett 333: 213221. https://doi.org/10.1016/j.canlet.2013.01.033

Murphy SK, Huang Z, Wen Y, Spillman MA, Whitaker RS, Simel LR, Nichols TD, Marks JR, Berchuck A (2006) Frequent IGF2/ H19 domain epigenetic alterations and elevated IGF2 expression in epithelial ovarian cancer. Mol Cancer Res 4: 283-292. https://doi. org/10.1158/1541-7786.MCR-05-0138

Phi LTH, Wijaya YT, Sari IN, Kim KS, Yang YG, Lee MW, Kwon HY (2019) 20(R)-Ginsenoside Rg3 influences cancer stem cell properties and the epithelial-mesenchymal transition in colorectal cancer via the SNAIL signaling axis. OncoTargets Ther 12: 10885-10895. https://doi.org/10.2147/O'T'T.S219063

Pisanic TR 2nd, Cope LM, Lin SF, Yen TT, Athamanolap P, Asaka R, Nakayama K, Fader AN, Wang TH, Shih IM, Wang TL (2018) Methylomic analysis of ovarian cancers identifies tumor-specific alterations readily detectable in early precursor lesions. Clin Cancer Res 24: 6536. https://doi.org/10.1158/1078-0432.CCR-18-1199

Tang M, Bian W, Cheng L, Zhang L, Jin R, Wang W, Zhang Y (2018) Ginsenoside Rg3 inhibits keloid fibroblast proliferation, angiogenesis and collagen synthesis in vitro via the TGF- $\beta /$ Smad and ERK signaling pathways. Int J Mol Med 41: 1487-1499. https://doi. org/10.3892/ijmm.2018.3362

Tian L, Shen D, Li X, Shan X, Wang X, Yan Q, Liu J (2016) Ginsenoside Rg3 inhibits epithelial-mesenchymal transition (EMT) and invasion of lung cancer by down-regulating FUT4. Oncotarget 7: 1619-1632. https://doi.org/10.18632/oncotarget.6451

Torre LA, Trabert B, DeSantis CE, Miller KD, Samimi G, Runowicz CD, Gaudet MM, Jemal A, Siegel RL (2018) Ovarian cancer statistics, 2018. CA Cancer J Clin 68: 284-296. https://doi.org/10.3322/ caac. 21456

Wang J, Tian L, Khan MN, Zhang L, Chen Q, Zhao Y, Yan Q, Fu L, Liu J (2018) Ginsenoside Rg3 sensitizes hypoxic lung cancer cells to cisplatin via blocking of NF- $x \mathrm{~B}$ mediated epithelial-mesenchymal transition and stemness. Cancer Lett 415: 73-85. https://doi. org/10.1016/j.canlet.2017.11.037
Wu Y, Zhou Y, He J, Sun H, Jin Z (2019) Long non-coding RNA H19 mediates ovarian cancer cell cisplatin-resistance and migration during EMT. Int J Clin Exp Pathol 12: 2506-2515. PMID: 31934077

Xie Q, Wen H, Zhang Q, Zhou W, Lin X, Xie D, Liu Y (2017) Inhibiting PI3K-AKt signaling pathway is involved in antitumor effects of ginsenoside Rg3 in lung cancer cell. Biomed Pharmacother 85: 16-21. https://doi.org/10.1016/j.biopha.2016.11.096

Yamaguchi H, Condeelis J (2007) Regulation of the actin cytoskeleton in cancer cell migration and invasion. Biochim Biophys Acta - Mol Cell Res 1773: 642-652. https://doi.org/10.1016/j.bbamcr.2006.07.001

Yokoi A, Matsuzaki J, Yamamoto Y, Yoneoka Y, Takahashi K, Shimizu H, Uehara T, Ishikawa M, Ikeda SI, Sonoda T, Kawauchi J, Takizawa S, Aoki Y, Niida S, Sakamoto H, Kato K, Kato T, Ochiya $\mathrm{T}$ (2018) Integrated extracellular microRNA profiling for ovarian cancer screening. Nat Commun 9: 4319. https://doi.org/10.1038/ s41467-018-06434-4

Yuan HD, Quan HY, Zhang Y, Kim SH, Chung SH (2010) 20 (S)Ginsenoside Rg3-induced apoptosis in HT-29 colon cancer cells is associated with AMPK signaling pathway. Mol Med Rep 3: 825-831. https://doi.org/10.3892/mmr.2010.328

Yuan Z, Jiang H, Zhu X, Liu X, Li J (2017) Ginsenoside Rg3 promotes cytotoxicity of Paclitaxel through inhibiting $\mathrm{NF}-x \mathrm{~B}$ signaling and regulating $\mathrm{Bax} / \mathrm{Bcl}-2$ expression on triple-negative breast cancer. Biomed Pharmacother 89: 227-232. https://doi.org/10.1016/j. biopha.2017.02.038

Zhao L, Shou H, Chen L, Gao W, Fang C, Zhang P (2019) Effects of ginsenoside $\mathrm{Rg} 3$ on epigenetic modification in ovarian cancer cells. Oncol Rep 41: 3209-3218. https://doi.org/10.3892/or.2019.7115

Zheng M, Hu Y, Gou R, Wang J, Nie X, Li X, Liu Q, Liu J, Lin $\mathrm{B}$ (2019) Integrated multi-omics analysis of genomics, epigenomics, and transcriptomics in ovarian carcinoma. Aging 11: 4198-4215. https://doi.org/10.18632/aging.102047

Zheng X, Chen W, Hou H, Li J, Li H, Sun X, Zhao L, Li X (2017) Ginsenoside $20(\mathrm{~S})-\operatorname{Rg} 3$ induced autophagy to inhibit migration and invasion of ovarian cancer. Biomed Pharmacother 85: 620-626. https://doi.org/10.1016/j.biopha.2016.11.072

Zheng X, Zhou Y, Chen W, Chen L, Lu J, He F, Li X, Zhao L (2018) Ginsenoside 20(S)-Rg3 prevents PKM2-targeting miR-324$5 \mathrm{p}$ from H19 sponging to antagonize the warburg effect in ovarian cancer cells. Cell Physiol Biochem 51: 1340-1353. https://doi. org $/ 10.1159 / 000495552$

Zheng ZG, Xu H, Suo SS, Xu XL, Ni MW, Gu LH, Chen W, Wang LY, Zhao Y, Tian B, Hua YJ (2016) The essential role of H19 contributing to cisplatin resistance by regulating glutathione metabolism in high-grade serous ovarian cancer. Sci Rep 6: 26093. https://doi. org/10.1038/srep26093

Zhou J, Yang L, Zhong T, Mueller M, Men Y, Zhang N, Xie J, Giang K, Chung H, Sun X, Lu L, Carmichael GG, Taylor HS, Huang Y (2015) H19 lncRNA alters DNA methylation genome wide by regulating S-adenosylhomocysteine hydrolase. Nat Commun 6: 10221. https://doi.org/10.1038/ncomms10221

Zou J, Su H, Zou C, Liang X, Fei Z (2020) Ginsenoside Rg3 suppresses the growth of gemcitabine-resistant pancreatic cancer cells by upregulating lncRNA-CASC2 and activating PTEN signaling. I Biochem Mol Toxicol e22480. https://doi.org/10.1002/jbt.22480 